\title{
Sammelbesprechung
}

\section{Vom Konkurrenten zum Untersuchungsgegenstand: Die Wirtschaftswissenschaften im Fokus (wirtschafts-) soziologischer Kritik}

Jochen Hörisch, Man muss dran glauben. Die Theologie der Märkte. München: Wilhelm Fink 2013, 132 S., kt., 18,90€

Giovanni Leghissa, Die Verfirmung der Gesellschaft. Zur Kritik des Neoliberalismus. München: Wilhelm Fink 2014, 104 S., kt., 13,90€

Jens Maeße (Hrsg.), Ökonomie, Diskurs, Regierung. Interdisziplinäre Perspektiven. Wiesbaden: Springer VS Verlag 2013, 321 S., br., 39,99€

Hanno Pahl / Jan Sparsam (Hrsg.), Wirtschaftswissenschaft als Oikodizee? Diskussionen im Anschluss an Joseph Vogls Gespenst des Kapitals. Wiesbaden: Springer VS Verlag 2013, 239 S., br., 29,99€

Jan Sparsam, Wirtschaft in der New Economic Sociology. Eine Systematisierung und Kritik. Wiesbaden: Springer VS 2015, 312 S., br., 49,99€

Sebastian Thieme, Der Ökonom als Menschenfeind? Über die misanthropischen Grundmuster der Ökonomik. Opladen: Barbara Budrich 2013, 103 S., br., 12,90€

Besprochen von Dr. Lisa Suckert: Max-Planck-Institut für Gesellschaftsforschung, Köln, E-Mail: suckert@mpifg.de

DOI 10.1515/srsr-2016-0050

Schlüsselwörter: Wirtschaftswissenschaften, Wirtschaftssoziologie, Performativität, Neoliberalismus

\section{Einleitung}

Der weltweite Erfolg der New Economic Sociology (NES) verdeutlicht es in aller Klarheit: Die einst von Talcott Parsons etablierte Arbeitsteilung zwischen Soziologie und Ökonomik ist Vergangenheit. Soziologen und Soziologinnen entdecken allerorts die Wirtschaft als ureigenen Untersuchungsgegenstand wieder. Für die NES ist der zentrale Ausgangspunkt, um sich mit Unternehmen, Märkten und Preisen auseinanderzusetzen, jedoch nicht (oder allenfalls oberflächlich) die 
Beschäftigung mit soziologischen Klassikern, die die Ökonomie seit jeher ins Zentrum ihrer Gesellschaftsanalysen gestellt haben. Stattdessen ist das neu erwachte Interesse für Wirtschaft von einer anderen Referenz geprägt, die Marion Fourcade pointiert zum Ausdruck bringt: „economic sociology constituted itself as that part of sociology that deals with the objects of economics, rather than economic objects broadly conceived“ (Fourcade, 2007: 1017).

Erstaunlich ist angesichts dieser Engführung auf die Untersuchungsgegenstände der Nachbardisziplin jedoch, dass die Neue Wirtschaftssoziologie sich zwar seit etwa drei Jahrzehnten an den Impotenzen der Wirtschaftswissenschaften abarbeitet, eine systematische Auseinandersetzung mit der Ökonomik und deren enger Verzahnung mit der Ökonomie aber weitestgehend unterlassen hat. ${ }^{1}$ Erst in jüngster Zeit versuchen insbesondere Arbeiten, die im Rahmen der Science Studies und/oder der Performativity-Ansätze zu verorten sind, diesen blinden Fleck der NES allmählich auszuleuchten.

Auch die hier zu besprechenden Neuerscheinungen setzen sich alle - implizit oder explizit - mit den Wirtschaftswissenschaften auseinander. Das Anliegen, die Ökonomik anders als die NES nicht nur als abstrakte Hintergrundfolie zu nutzen, sondern einer fundierten Kritik zu unterziehen, wird so im deutschsprachigen Raum aufgegriffen und fruchtbar erweitert. Im Folgenden werden mit einem von Jens Maeße herausgegebenen Sammelband und den Essays von Giovanni Leghissa und Jochen Hörisch zunächst drei Bücher vorgestellt, die sich mit der Regierung von und durch die Wirtschaft befassen und dabei nicht zuletzt auf die zentrale Rolle der Ökonomik für die Verfasstheit des ökonomischen Systems eingehen. Der Sammelband von Jan Sparsam und Hanno Pahl sowie die Abhandlung von Sebastian Thieme stellen demgegenüber die Wirtschaftswissenschaften selbst in den Mittelpunkt und durchleuchten deren Grundannahmen, Methodologie und Leerstellen äußerst kritisch. Die umfassende Analyse von Jan Sparsam setzt sich ebenfalls mit den Mängeln der Ökonomik auseinander, hinterfragt aber auch die Versuche der NES kritisch, sich von der wirtschaftswissenschaftlichen Perspektive abzusetzen. Abschließend werden gemeinsame Tendenzen und Desiderate der rezensierten Bücher in einem Fazit herausgearbeitet.

1 Vorab sei angemerkt, dass in den besprochenen Bänden Begriffe wie Ökonomie, Ökonomik, VWL und Wirtschaftswissenschaften höchst uneinheitlich verwendet werden. Im Rahmen dieser Besprechung soll die Bezeichnung „Ökonomik“ die Wissenschaft von der Ökonomie benennen und als Oberbegriff der beiden Wirtschaftswissenschaften BWL und VWL gelten. 


\section{Wirtschaftswissenschaften und ökonomische Regierung}

Der von Jens Maeße herausgegebene Sammelband „Ökonomie, Diskurs, Regierung“ widmet sich dem Verhältnis von Diskurs und Ökonomie und beleuchtet insbesondere die hegemoniale Stellung des wirtschaftswissenschaftlichen Diskurses. In seiner Einleitung positioniert Maeße die Beiträge im weiteren Forschungsfeld: Sie knüpfen sowohl an die oben beschriebene Wiederentdeckung des Ökonomischen durch die Soziologie als auch an die zunehmende Bedeutung von Diskursivität in vielen Forschungsprogrammen an. Neben Einblicken in die vielseitige deutschsprachige Forschung enthält der Band die Übersetzungen zweier prominenter Artikel: einen programmatischen Aufsatz von Marion Fourcade zum Verhältnis von Wirtschaftssoziologie und Gesellschaftstheorie aus dem Jahr 2007 und eine Studie von Frederic Lebaron aus dem Jahr 2006, die sich mit dem symbolischen Kapital von ökonomischen Nobelpreisträgern auseinandersetzt. Da beide Artikel bereits vielfach Beachtung gefunden haben, werden sie an dieser Stelle nicht vertieft.

Der Sammelband gliedert sich in drei zentrale Kapitel. Die Beiträge des ersten Kapitels beleuchten die Regulierung wirtschaftlicher Ordnung und zeigen auf, inwieweit hier wirtschaftswissenschaftliche Diskurse eine Rolle spielen. In einem ersten Beitrag zeigt Oliver Kessler die engen Grenzen des Diskurses zur aktuellen Finanzmarktkrise auf. Es wird deutlich, dass durch die Dominanz und die restriktiven Vorannahmen wirtschaftswissenschaftlicher Modelle alternative Expertisen systematisch ausgeschlossen werden. Stattdessen werden Krisenursachen externalisiert (der Staat, der nicht reguliert hat) und der Mythos der Effizienzmarkthypothese (die Krise als Fehler, der aber außerhalb des Systems liegt) perpetuiert. Der Aufsatz von Benjamin Wilhelm versucht sich am Entwurf einer politischen Ökonomie der Finanzmarktregulierung. Es wird skizziert, wie sich der Regulierungsdiskurs in Zusammenhang mit den Basel-Abkommen an der Schnittstelle zwischen politischer und ökonomischer Logik entfaltet. Amelie Kutter belegt anhand von journalistischen Artikeln, in denen „Lehren“ aus der aktuellen Wirtschaftskrise gezogen werden, dass die gegenwärtige Krise bislang keinesfalls zu einem ökonomischen Paradigmenwechsel geführt hat. Zwar wurden alternative wirtschaftswissenschaftliche Diskurse zur Rolle des Staates vereinnahmt, die neoliberale Deutungshoheit bleibt jedoch erhalten. Der weiterführenden Frage, warum alternative ökonomische Perspektiven derart leicht kooptiert werden können, geht Kutters äußerst gelungene Analyse jedoch nicht nach.

Im zweiten Kapitel sind Beiträge zusammengefasst, die untersuchen, auf welche Art und Weise ökonomische Konzepte in öffentlichen Diskursen auf- 
genommen und dort prozessiert werden. Der Beitrag von Alexander Ziem zeichnet methodisch äußerst versiert nach, wie das ökonomische Phänomen Finanzkrise in medial vermittelten Diskursen anonymisiert und zur Naturgewalt umgedeutet wird. Menschen werden im Zusammenhang mit der Krise zwar als Betroffene thematisiert, als Urheber des ökonomischen Systems jedoch ausgeblendet. Weiterführend wäre eine stärkere Reflexion der Ursachen und Implikationen dieser Naturalisierung, insbesondere im Hinblick auf die hier thematisierten Wirtschaftswissenschaften, wünschenswert. Der Aufsatz von Ronny Scholz basiert auf dem gleichen Projekt wie Ziems Beitrag, beleuchtet aber die Frage, ob im Rahmen der medialen Krisendebatte eine Verschiebung hinsichtlich der als legitim wahrgenommenen politischen Handlungsträger stattgefunden hat. Scholz zeigt, dass die Ursachen der Krise zwar als transnational dargestellt werden, die Folgenbekämpfung aber weiterhin dem Nationalstaat zugeschrieben wird. Während die Beiträge von Ziem und Scholz auf eine Anonymisierung von ökonomischen Krisenursachen abheben, kommt Jan Krasni zum entgegengesetzten Befund und zeigt auf, wie Ursachen in Fernseh- und Online-Beiträgen personalisiert werden. Krasni analysiert dabei insbesondere, wie ökonomische Sachverhalte und wirtschaftswissenschaftliche Instrumente (z.B. Kurvenverläufe) visuell inszeniert werden und sich dadurch als Allgemeinwissen ins kollektive Gedächtnis einprägen.

Das letzte Kapitel betrachtet schließlich das akademische Feld der Ökonomik als diskursive Arena und zeigt auf, unter welchen Bedingungen Wissen über die Ökonomie erzeugt wird. Gertraude Krell vollzieht in ihrem als Inspektion deklarierten und durch Foucault inspirierten Essay nach, wie die BWL ihren Untersuchungsgegenstand konstruiert. Indem Wirtschaft auf die Phänomene Markt und Unternehmen beschränkt wird, werden private Haushalte zu Orten des NichtWirtschaftens degradiert. Krell zeigt die genderpolitischen Implikationen dieser Ausrichtung auf und deutet die Folgen einer kapitalistischen Transformation hin zu einem „neuen Geist des Kapitalismus“ an. Jens Maeße zeichnet nach, wie sich das Feld der Ökonomik im Wechselspiel zwischen wirtschaftsnaher BWL und staatsformender VWL sowie zwischen öffentlichkeitswirksamer Debatte und der reinen akademischen Welt historisch etabliert hat. Analog zur institutionellen Heterogenität dieser vier Pole wurde Multireferentialität, d.h. die Fähigkeit, eigene Diskursbeiträge mehrdeutig zu gestalten, zu einer wichtigen diskursiven Ressource. Da Maeße viele Argumentationsfiguren auf begrenztem Raum verdichtet, sind die kategorialen Oppositionen (vier Pole, horizontal vs. vertikal, rechts vs. links, Fürsprecher vs. Gegenspieler) für den Leser nicht immer klar nachzuvollziehen. Es gelingt ihm aber gerade deswegen, ein umfassendes Bild der Ökonomik als einen Ort der heterogenen Wissensproduktion zu zeichnen. Der Beitrag von Hanno Pahl betrachtet die Rolle von Metaphern für die wirtschaftswissen- 
schaftliche Theoriebildung. Metaphern ermöglichen der Ökonomik einerseits, ihren abstrakten Erkenntnisgegenstand und sich selbst als Disziplin zu erfassen, und andererseits, ihre Theorien im Rahmen von gesellschaftlichen Debatten konkretisier- und damit vermittelbar zu machen. Es zeigt sich jedoch, dass insbesondere jene Metaphern, auf denen das hegemoniale neoklassische Paradigma beruht (unsichtbare Hand, Gleichgewicht und Tâtonnement), immer wieder umgedeutet wurden. Ähnliches skizziert Pahl für die Konzeption von ökonomischen Lehrbüchern. Während Pahl mit seiner Analyse ausschließlich die VWL ins Visier nimmt, wendet sich der abschließende Aufsatz von Alexander Preisinger der Managementmethode des Storytelling zu, die „Erzählen“ instrumentalisiert, um Wissen effizienter und überzeugender zu vermitteln. Basierend auf der Analyse von Storytelling-Ratgebern wird aufgezeigt, wie im Kern strukturalistische Analyseinstrumente durch die BWL-Literatur vereinnahmt, einem Mittel-Zweck-Kalkül unterworfen und zu Management-Tools umgedeutet werden. Dies, so attestiert Preisinger, geht jedoch nicht ohne erhebliche Paradoxien vonstatten.

Der Sammelband wird durch sehr unterschiedliche Zugänge, auch gerade $\mathrm{zu}$ den Zentralreferenzen „Ökonomie“ und „Diskurs“, geprägt. Dies ist sicher nicht zuletzt darauf zurückzuführen, dass die meisten Beiträge eher im Überlappungsbereich verschiedener Fachrichtungen zu verorten sind. Gerade aus dieser Pluralität ergeben sich jedoch erfrischende und inspirierende Perspektiven auf das Phänomen. Die von Maeße sehr gelungen zusammengestellten Beiträge zeigen, auf welch vielfältige Weise die diskursive Dominanz der Ökonomik das legitime Verhältnis von Ökonomie und Regierung prägt. Da der Band, en passant, auch ein sehr breites methodisch-diskursanalytisches Spektrum abdeckt, eignet er sich vorzüglich für all jene, die sich überblicksartig mit der Diskursivität von Ökonomie auseinandersetzen und dieses wichtige Forschungsfeld für sich erschließen möchten.

Das kompakte Büchlein von Giovanni Leghissa macht es sich zur Aufgabe, die „Verfirmung der Gesellschaft“ zu analysieren. Unter „Verfirmung“ versteht Leghissa, dass Regeln und Herrschaftstechniken, die ursprünglich in Unternehmen Anwendung gefunden haben, zunehmend zur legitimen Steuerung anderer Gesellschaftsbereiche eingesetzt werden. Um diesen Befund in einen größeren Rahmen einzuordnen, versucht sich der Band an einer breiter angelegten Kritik des Neoliberalismus und den damit einhergehenden neoliberalen Subjektivierungsformen. In einem ersten Kapitel wird hierzu die Foucault'sche Perspektive und insbesondere die Differenzierung zwischen Liberalismus und Neoliberalismus als theoretischer Anker herausgearbeitet. Das neoliberale Projekt versteht den Markt nicht nur als Höhepunkt der ökonomischen Evolution, sondern erhebt das Kosten-Nutzen-Kalkül zur universellen Norm, nach der alle Gesellschaftsbereiche inklusive der Politik zu gestalten sind. Indem Effizienz zum normativen Selbst- 
zweck erhoben wird, so Leghissas zentrales Argument, wird jede menschliche Unternehmung und am Ende der Mensch selbst im Lichte der idealtypischen Schablone „Unternehmen“ bewertet. Das zweite Kapitel gibt Einblicke in die zeitgenössische Gouvernementalitätsforschung und stellt klar, dass Neoliberalismus nicht als Rückzug des Staates, sondern vielmehr als ein Implementieren neoliberaler Kontrolltechniken durch den Staat zu verstehen ist. Im dritten Kapitel erfolgt schließlich eine umfassende Kritik am Rationalitätsbegriff neoliberaler Theorien - hiermit ist der ökonomische Mainstream gemeint - und eine Gegenüberstellung mit alternativen wirtschaftswissenschaftlichen Zugängen. Dabei arbeitet Leghissa sehr gekonnt die enge Verbindung zwischen Rational Choice und biologischen Evolutionstheorien heraus. Das vierte Kapitel stellt schließlich den Kern der Abhandlung dar, in dem ,die biopolitische Tragweite der betrieblichen Praktiken“ aufgegriffen wird. Leghissa argumentiert, dass die betriebswirtschaftliche Organisationstheorie (und hier maßgeblich der von Herbert Simon geprägte Zugang), obgleich sie sich als Alternative zur neoklassischen VWL inszeniert, maßgeblich zur praktischen Verwirklichung des neoliberalen Rationalitätsmodells beiträgt. Die Organisationslehre etabliert eine Perspektive, die dazu aufruft, nicht-ökonomische Sphären (das Soziale, das Biologische, das Politische) $\mathrm{zu}$ instrumentalisieren, um das Ökonomische zu optimieren. Diese Herangehensweise hat aber nicht nur Auswirkungen auf die Arbeitsbedingungen innerhalb der Organisation, sondern dient auch als Modell für eine weitergehende neoliberale Subjektivierung. Individuen werden dazu angehalten, sich selbst als Unternehmen zu begreifen, ihre eigenen körperlichen Besonderheiten, Fähigkeiten, Charakterzüge und Motive als Ressourcen zu verstehen und entsprechend $\mathrm{zu}$ optimieren.

Das schmale Bändlein nimmt sich mit dem Neoliberalismus zugegebenermaßen eines der größten und diffusesten Themen der Gegenwart an. Es ist sicher auch auf diesen Umstand zurückzuführen, dass die einzelnen Argumente häufig mit schnellem Strich entwickelt werden und nicht immer ausreichend in die Tiefe greifen. Insbesondere kommt aber der titelgebende Begriff der „Verfirmung“ zu kurz. Außer dem pauschalen Befund, dass Rationalitätslogiken für alle Lebensbereiche und Organisationsformen zunehmend Geltung erfahren, findet sich wenig dazu, wie eine etwaige „Verfirmung“ als Prozess, vermittelt über die Organisationstheorie, voranschreitet, durch welche Faktoren sie begünstigt wird, welche konkreten und vielfältigen Formen sie annimmt oder auf welche Widerständigkeiten des Sozialen sie trifft. Nichtsdestotrotz macht Leghissa das Foucault'sche Instrumentarium gekonnt nutzbar, um die Managementtheorie kritisch $\mathrm{zu}$ beleuchten. Seine Abhandlung weist eindrücklich darauf hin, dass auch die Organisationslehre, die sich selbst häufig den Anstrich gibt, humanistische Momente zu integrieren, als Implementationstechnologie im Sinne einer durch und 
durch neoliberalen Subjektivierung verstanden werden sollte - und zeigt damit eine Forschungslücke auf.

Jochen Hörisch möchte seinen Band „Man muss dran glauben“ von vorneherein als Traktat verstanden wissen. Diesem Anspruch wird das Buch gleich im Doppelsinn des Wortes gerecht. So handelt es sich einerseits um eine kurze wissenschaftliche Erörterung, die die enge Verwandtschaft von Ökonomie und Religion beleuchtet, aber eben andererseits auch um eine sehr deutliche Streitund Schmähschrift. Wer hier geschmäht werden soll, wird entlang Hörischs Argument schnell deutlich: Es sind die Wirtschaftswissenschaften, die „Priesterklasse der neoliberalen Wirtschaftsweisen“, die ähnlich der Theologie versuchen, ökonomische Phänomene zu deuten. Während die Theologie sich aber im Rahmen der Aufklärung gewandelt hat und die Religion heute kritisch hinterfragt, wurde jegliche ökonomische Aufklärung bislang verschlafen. Der zentrale Vorwurf an die Ökonomik lautet, dass sie nicht zu einer reflektierten Auseinandersetzung mit ihrem Untersuchungsgegenstand in der Lage ist.

Um die religiöse Qualität kapitalistischen Wirtschaftens und die theologischen Züge der neoklassischen Ökonomik darzulegen, werden die zentralen Argumente des Traktats zunächst in acht Kapiteln entwickelt, die die Parallelen zwischen Ökonomie und Religion aus unterschiedlichen Perspektiven beleuchten. So zeigt Hörisch immer wieder auf, dass sowohl Geld als auch Gott nur existieren, solange an sie geglaubt wird. Parallelen macht Hörisch aber auch im Hinblick auf die Kontingenzbewältigung aus: Um eine (ökonomische) Welt voller Paradoxien und Kontingenz zu ertragen, bedarf der Mensch einer ultimativen Größe, die diese Widersprüche zumindest vordergründig auflöst. Was dem Gläubigen Gott, ist dem ökonomischen Akteur und nicht zuletzt dem Ökonomen der Markt. So formuliert Hörisch zynisch: „Unerforschlich wie der Ratschluss Gottes ist der Ratschluss des Marktes, mächtig wie die Hand Gottes führt uns die invisible hand des Marktes durch Turbulenzen, deren Sinn wir nicht verstehen können“ (51). Auch der Zusammenhang von Schuld und Tugend wird in der modernen Ökonomik ähnlich wie in der Religion konzipiert: Im Rahmen der neoklassischen Gleichgewichtstheorie werden Egoismus und „kreative“ Zerstörung überhöht und als Quelle für ein übergeordnetes Gemeinwohl dargestellt. Kurz: Es braucht das Böse, damit das Gute walten kann. Schließlich verdeutlicht Hörisch an zahlreichen Beispielen die transzendente Natur von Geld, die auch das Göttliche charakterisiert. Geld kann die Form von unendlich vielen Waren und Dienstleistungen annehmen und transzendiert zudem die Zeit: Es speichert den Wert aus vergangener Arbeit für die Zukunft und überträgt Werte aus der Zukunft mittels Zinsen in die Gegenwart. Ausgehend von diesen Parallelen werden im neunten Kapitel konkrete Vorschläge für eine ökonomische Aufklärung gemacht. Da entlang der Abhandlung jede positive Assoziation mit Planwirtschaft oder 
Sowjetmarxismus entschieden und fast schon ein wenig reflexhaft zurückgewiesen wird, erscheinen die Vorschläge Hörischs wenig revolutionär und reichen von einer Erbschaftssteuer über die Begrenzung von Bonuszahlungen bis zur Risikobindung von Gewinn. In der Zusammenschau liest sich dieses Handlungsprogramm aber dennoch instruktiv und könnte immerhin einen gesamtgesellschaftlichen (Minimal-)Konsens erzeugen. Der Band wird abschließend durch zwei kurze Texte ergänzt, die Argumentationsstränge des Traktats aufgreifen und anhand der Werke von Johann Wolfgang von Goethe und Thomas Mann erörtern.

Die Abhandlung, die mit viel Wortwitz, eingängig und eindringlich formuliert ist und immer wieder pointierte Bezüge zur aktuellen Wirtschafts- und Finanzkrise verwebt, unterscheidet sich von den übrigen hier vorgestellten Büchern nicht zuletzt durch die disziplinäre Herkunft des Autors, eines Literaturwissenschaftlers. Es ist sicher auch der „Fachfremde“ des Autors geschuldet, dass das Traktat jede Menge erfrischende Einsichten und überraschende Perspektiven aufweist. Für sozialwissenschaftliche Leserinnen erscheint es hingegen gewöhnungsbedürftig, wenn Thesen nicht durch empirische Bezüge oder eine theoretische Herleitung, sondern durch Zitate aus literarischen Werken belegt werden. Auch die Vehemenz, mit der die semantischen Ähnlichkeiten zwischen religiöser und ökonomischer Sphäre ausgemacht werden (z. B. im Bezug auf die Trinität des Geldes), erscheinen streckenweise etwas bemüht. Hervorragende Überzeugungskraft entfaltet Hörischs Argument immer dann, wenn er strukturelle und funktionelle Parallelen aufzeigt, die zwar von der Ebene der Semantik gestützt werden, aber doch über diese hinaus gehen. Insgesamt liefert „Man muss dran glauben“ entscheidende Denkanstöße für ein Thema mit großer Brisanz und ermutigt, im Anschluss an die Klassiker der Soziologie, die Verwandtschaft von Ökonomie und Religion wieder stärker in den Fokus zu stellen und darüber hinaus die Parallelen zwischen Ökonomik und Theologie kritisch zu beleuchten.

\section{Wirtschaftswissenschaften auf dem soziologischen Prüfstand}

Auf Analogien zwischen der Ökonomik und der religiösen Sphäre verweist auch das Konzept der „Oikodizee“, das im Zentrum des von Hanno Pahl und Jan Sparsam herausgegebenen Sammelbandes „Wirtschaftswissenschaft als Oikodizee?“ steht. Unter Oikodizee wird hier ein fundamentaler Glaube an die Allmacht und Legitimität des Marktes verstanden, der sich weder durch moralische Kritik noch durch empirische Befunde beirren lässt. Mit dieser Zentralreferenz verweist der Sammelband, wie der Untertitel bereits andeutet, auf das Buch „Das Gespenst des 
Kapitals“ von Joseph Vogl (2010). Vogls Konzept der Oikodizee bildet den Ausgangspunkt aller Beiträge, die sich in teils kritischer, teils konstruktiver Weise mit dem Zustand der Wirtschaftswissenschaften in Zeiten der Krise auseinandersetzen. In ihrer Einleitung legen die beiden Herausgeber überzeugend dar, warum eine solche Beschäftigung mit der Ökonomik nottut: Zwar ist eine pauschale und oft zynische Kritik an den Wirtschaftswissenschaften durchaus in Mode geraten, in der Ökonomik selbst ist diese Kritik jedoch größtenteils folgenlos verhallt. Das neoklassische Paradigma dominiert die Disziplin weiter unangefochten. Der Sammelband möchte daher nicht nur zu einer intensiveren, kritischen Kommunikation über, sondern auch mit den Wirtschaftswissenschaften anregen.

Die zehn Beiträge des Sammelbandes, von denen hier lediglich acht besprochen werden, ${ }^{2}$ sind in drei thematische Schwerpunkte untergliedert. Das erste Kapitel beschäftigt sich mit dem dominanten Paradigma der Neoklassik und vertieft Argumente, die Vogl in seinem Buch hierzu skizziert. Der Beitrag von Verena Halsmayer und Florian Huber ordnet das Konzept der Oikodizee in das weitere Werk von Joseph Vogl ein. Die beiden Autoren vollziehen die These der Oikodizee anhand von zwei Beispielen der ökonomischen Wissensproduktion nach. Ihr Befund deckt sich zwar in Teilen mit Vogls Argument, sie werfen ihm jedoch eine zu starke Vereinfachung auf den neoklassischen Kanon vor. Zudem bemängeln sie, dass sich Vogl, entgegen eigenen Vorarbeiten, allein auf textliche Quellen fokussiert und so unterschiedliche Forschungs-, Darstellungs- und Formalisierungspraktiken aus dem Bild geraten. Ivan Boldyrev stellt in seinem Beitrag die Performativität der Wirtschaftswissenschaften in den Mittelpunkt, einen Aspekt, der auch bei Vogl zentral ist. Am Beispiel der (marktsozialistischen) Gleichgewichtstheorie zeigt Boldyrev auf, wie ökonomische Theorien gesellschaftliche Wirkung entfalten. Die Crux besteht einerseits darin, zu untersuchen, wie ökonomische Theorie und Praxis verschränkt sind. Andererseits gilt es aber auch aufzuzeigen, wie sie von Ökonomen aktiv verschränkt werden und inwiefern ökonomische Modelle bereits so angelegt sind, dass sie eine derartige Performativität begünstigen. Der wissenschaftskritische Aufsatz von Till Düppe geht der These Vogls nach, dass Ökonomik im Kern eine als Wissenschaft verkleidete Ideologie sei, die Machtinteressen durchsetzt. Mit einem historischen Abriss über die US-amerikanischen Institutionalisierungsbedingungen versucht er zu zeigen, dass Stil und Methodologie der Ökonomik im Gegenteil aus dem Bedürfnis entstanden sind, sich zu verstecken, um, paradoxerweise, nicht als sozialistisch

2 Die Beiträge der beiden Herausgeber Hanno Pahl und Jan Sparsam werden hier nicht dezidiert vorgestellt, da sich die zentralen Argumentationsstränge ihrer Artikel bereits andernorts in dieser Rezension finden. Beiden Autoren gelingt es sehr überzeugend, ihre Ideen mit dem Konzept der Oikodizee zu verbinden und entsprechend weiterzuentwickeln. 
enttarnt zu werden. In der einhergehenden Diskursfeindlichkeit sieht Düppe den Beleg dafür, dass Ökonomik per se nicht reformierbar ist, und empfiehlt deren Auflösung. Unklar bleibt allerdings, wie und warum sich aus einer sozialistischen Grundhaltung - quasi im Versteck der Formalisierung - der häufig attestierte neoliberale Bias der Wirtschaftswissenschaften entwickeln konnte.

Die Beiträge des zweiten Kapitels nehmen Alternativen zur neoklassischen Theorie ins Visier, die den ökonomischen Mainstream hinterfragen. Bernd Bösel und Manuel Wäckerle fokussieren sich auf den Aspekt der Zeitlichkeit in Vogls Argument. In Auseinandersetzung mit der poststrukturalistischen Ereignisphilosophie wird aufgezeigt, dass neoklassische Modelle im Kern darauf zielen, den Ereignischarakter der Zeit durch ökonomische Prognosetechniken zu bändigen. Tatsächliche Krisenereignisse müssen dieser Ökonomik daher als „gespenstisch“ erscheinen, als etwas, das es nicht geben kann. Die Autoren fordern daher eine alternative Ökonomik, die sich vor Krisenereignissen nicht fürchtet, sondern, wie z.B. evolutorisch-institutionelle Ansätze versucht, diese durch eine historische Kontextanalyse zu erklären. Der Beitrag von Christoph Deutschmann schließt an die von Vogl hervorgebrachte Kritik der Finanzmärkte an, hebt allerdings hervor, dass es nicht nur einer realistischeren Modellierung der Finanzmärkte bedarf, sondern auch einer Perspektive, die langfristige ökonomische Strukturverschiebungen im Sinne einer Finanzialisierung aufdeckt. Um den Aufstieg des Finanzsektors theoretisch greifbar zu machen, schlägt er vor, weltsystemtheoretische Ansätze, die Finanzialisierung als Phase des Niedergangs der kapitalistischen Hegemonie deuten, mit einer Mehrebenenerklärung zu fundieren. Ausgehend von diesem Modell skizziert Deutschmann anschaulich, wie der Niedergang des klassischen Unternehmertums zur anhaltenden Finanzialisierung beigetragen hat. Dabei wurden unternehmerische Potentiale auf der Meso- und Mikro-Ebene sukzessive abgebaut.

Das dritte Kapitel wendet sich schließlich der Ökonomik-Kritik selbst zu. Remigius Bunia beschäftigt sich mit Vogls ablehnender Haltung gegenüber Finanzmarktspekulation. Während Spekulation heute maßgeblich ökonomisch gedeutet und dafür kritisiert wird, dem Glauben aufzusitzen, man könne die Zukunft rational berechnen, verbirgt sich hinter dem Begriff auch ein philosophisches Konzept. Spekulatives Denken, so Bunia, gerate aber gerade durch den um sich greifenden Rationalismus in Verruf. Bunia appelliert daher für mehr Spekulation im Sinne eines Denkens, das sich nicht über die Methoden der jeweiligen Disziplin absichern muss, und sieht Vogls Auseinandersetzung mit dem Kapitalismus als wertvolles Beispiel hierfür. Deutlich kritischer setzt sich Simon Deichsel mit Vogls Thesen zur (Ir-)Rationaliät der Ökonomik auseinander. Er wirft Vogl einerseits vor, mit einem kaum bestimmten Konzept von Rationalität zu operieren (was Deichsel allerdings dazu verleitet, Vogl ein allzu ökonomisches Verständnis 
von Rationalität $\mathrm{zu}$ unterstellen) und andererseits, neuere Entwicklungen der Institutionenökonomik nicht mit einzubeziehen. Nur so könne Vogl das Bild einer Ökonomik, die die unerwünschten Effekte von eigennützigem Handeln und die Unzulänglichkeiten des „freien Marktes“ ignoriert, aufrechterhalten. Ebenfalls kritisch durchleuchtet Roman Köster die Art und Weise, wie Vogl die Entwicklung der Ökonomik historisch nachzeichnet. Er wirft Vogl vor, die Genealogie äußerst lückenhaft und einseitig darzustellen, um sein eigenes Argument zu plausibilisieren. Als tatsächlich problematisch erscheint Köster nicht so sehr, dass die Ökonomik falsche Vorhersagen macht, sondern das utopische gesellschaftliche Bedürfnis nach Prognosen, das die Ökonomik unreflektiert bedient.

Das von Hanno Pahl und Jan Sparsam herausgegebene Buch bietet eine sehr vielseitige und in weiten Teilen konstruktive Auseinandersetzung mit den Mängeln der Wirtschaftswissenschaften. Der gemeinsame Bezugspunkt aller Beiträge - das Buch Vogls und dessen zentrale Konzepte - verhindert, dass der Sammelband als Aneinanderreihung einzelner Artikel erscheint. Eine vorherige Auseinandersetzung mit Vogls Abhandlung ist dabei jedoch nicht zwingend notwendig. Den Herausgebern ist ein abwechslungsreicher, aber konzentrierter Band gelungen, der lesenswert erscheint für alle an der Ökonomik Interessierten und nicht zuletzt für Wirtschaftswissenschaftler, die ihre eigene Disziplin neu und anders entdecken wollen.

Eine innovative Perspektive gegenüber den Wirtschaftswissenschaften nimmt auch das Buch „Der Ökonom als Menschenfeind?“ von Sebastian Thieme ein, in dem der provokanten Frage nachgegangen wird, inwiefern die Ökonomik als misanthropisch gelten muss. Insbesondere geht es Thieme darum aufzuzeigen, inwiefern eine gewisse Menschenfeindlichkeit, die Menschen in nützlich und weniger nützlich unterscheidet, bereits im Kern der ökonomischen Theorie angelegt ist.

Hierzu untersucht Thieme sowohl die Aussagen einzelner Wirtschaftswissenschaftler als auch die Grundpfeiler neoklassischer und institutionenökonomischer Theorieschulen im Hinblick auf menschenfeindliche Elemente und arbeitet sukzessive drei zentrale Aspekte heraus: Er kann erstens nachzeichnen, dass den untersuchten Theorien ein durchweg negatives Menschenbild zugrundeliegt. Der Mensch wird per se als arbeitsavers, opportunistisch und eigennützig angenommen. Zweitens wird der Markt als theoretisches Konzept entlarvt, das die natürliche Auslese und den damit verbundenen Daseinskampf zur einzigen Tugend stilisiert. Wettbewerb, so Thieme, impliziert zwangsläufig die Erzeugung von Ungleichwertigkeiten, d.h. von Gewinnern und Verlierern. Der Mitmensch, den man als gleichen schätzt, wird so zum erbitterten Konkurrenten, den es auszustechen gilt. Drittens zeigt Thieme, wie der hohe Abstraktionsgrad der Wirtschaftswissenschaften dazu beiträgt, etwaige misanthropische Tendenzen hinter einer scheinbaren „Objektivität“ zu verschleiern. Zudem bewirkt der hohe Forma- 
lisierungsgrad eine weitere Verdinglichung und damit „Entmenschlichung“. Ausgehend von der entlang der Analyse ökonomischer Grundlagentexte gewonnenen Heuristik wendet sich Thieme aktuellen wirtschaftspolitischen Zusammenhängen und den entsprechenden Empfehlungen von Wirtschaftswissenschaftlern zu. Dabei beleuchtet er verschiedene Einschätzungen zur Arbeitsmarktpolitik, Empfehlungen zur Vermeidung von betrieblichem Diebstahl und Gary Beckers auf Kosten-Nutzen-Erwägungen basierendes Plädoyer für die Todesstrafe. Abschließend wird aufgezeigt, in welchem Maße abwertende Stereotype tatsächlich von Ökonomen vorgebracht und in Politik und Medien aufgegriffen werden. Hierbei gelingt es, die drei oben genannten Komponenten mit aktuellem empirischem Anschauungsmaterial zu unterfüttern. Es wird so deutlich, dass die untersuchten wirtschaftswissenschaftlichen Empfehlungen in ihren Annahmen, aber auch in ihrer intendierten Wirkung, durchaus als menschenfeindlich bezeichnet werden können. Sebastian Thieme, der selbst Volkswirt ist, belässt es aber nicht bei diesem desaströsen Befund für die Wirtschaftswissenschaften, sondern beharrt darauf, dass Ökonomik und Ethik nicht per se inkompatibel sind. Anhand einer Reihe von Theoretikern, die ein anderes Menschenbild und eine andere Funktionsweise von Wirtschaft angemahnt haben, zeigt er - angefangen bei Adam Smith, dem Urvater der Ökonomik -, dass der Perspektivenwechsel, d.h. sich in den Mitmenschen hineinversetzen, und ein öffentlicher Diskurs über Zumutbarkeit und Verhältnismäßigkeit wichtige Elemente zur Eindämmung menschenfeindlicher Tendenzen in der Ökonomik sein könnten. Darauf aufbauend werden in einer Zusammenfassung weitere Forschungsfragen und Desiderate skizziert.

Gleich in seiner Einleitung verweist Thieme darauf, dass seine Abhandlung eher einen Aufriss des Problems denn eine detaillierte Analyse darstellt. Der kursorische Charakter des Werkes ist somit explizit intendiert, führt jedoch, z. B. wenn der vielversprechende Aspekt der Verdinglichung nur oberflächlich gestreift wird, zum Wunsch nach Vertiefung. Der Skizzenhaftigkeit des Bandes mag auch geschuldet sein, dass Begrifflichkeiten nicht immer in aller Schärfe ausdefiniert werden. So wird der Begriff der Menschenfeindlichkeit zwar mit Verweisen auf empirische Untersuchungen und vielen Beispielen untermalt, für die eigene Untersuchung aber nicht klar theoretisch umrissen. Es bleibt unklar, was eine ökonomische Menschenfeindlichkeit im Sinne einer Verschiedenwertigkeit von anderen Formen der Abwertung, die im Einzelfall durchaus als legitim erachtet werden können (z.B. die Stigmatisierung von Rassisten oder Homophoben), abhebt. Nichtsdestotrotz gelingt es Thieme sehr eindrucksvoll und in pointiertem Stil aufzuzeigen, auf welch verdeckten normativen und, wie er überzeugend darlegt, menschenfeindlichen Annahmen die gegenwärtige Ökonomik beruht. Es ist sein Verdienst, diese wichtigen Fragen erstmals aufzuwerfen und darüber hinaus einen inspirierenden Reformhorizont für die Wirtschaftswissenschaften zu skizzieren. 
Die metatheoretische Analyse von Jan Sparsam greift viele der bis hierher dargestellten Kritikpunkte an der Ökonomik auf. Tatsächlich richtet sich Sparsams Augenmerk aber nicht auf die Ökonomen, sondern explizit auf jenen Forschungsstrang, der sich als zentraler Antagonist der Wirtschaftswissenschaften inszeniert: die New Economic Sociology. Das Buch „Wirtschaft in der New Economic Sociology“ überprüft die Theorien der NES auf Konsistenz und Leerstellen und geht der Frage nach, wie die Wirtschaftssoziologie im Unterschied zur Ökonomik „das Ökonomische“ als ihren Gegenstand konzipiert. Der zentrale Vorwurf an die NES wird schon im einleitenden Kapitel formuliert: nämlich dass die Wirtschaftssoziologie unhinterfragte Annahmen der Ökonomik hinsichtlich des kapitalistischen Systems übernimmt und damit die Fehler der Ökonomik fortschreibt. Um diesen Vorwurf zu fundieren, entwickelt Sparsam zunächst im zweiten Kapitel ein anspruchsvolles metatheoretisches Analyseraster, das sich maßgeblich auf Konzepte der Wissenschaftssoziologie und auf die kritische Soziologie Adornos stützt. Das dritte Kapitel zeichnet anschließend die institutionellen Entstehungsbedingungen und paradigmatischen Grundannahmen der NES nach. Sparsam macht deutlich, dass die NES sich allenfalls oberflächlich legitimatorisch auf (wirtschafts-)soziologische Klassiker bezieht. Stattdessen speist sich der Aufstieg der NES nicht zuletzt aus dem Niedergang der Differenzierungstheorie: Verdeutlicht doch die Zentralreferenz der „Einbettung“ gerade den Anspruch, Wirtschaft als Teilbereich der Gesellschaft zu betrachten. Als weiteren zentralen Einfluss stellt Sparsam die enormen wirtschaftlichen Umbrüche seit den 1970er Jahren und die Herausbildung eines neu- und andersartigen Kapitalismus heraus. Diese hätten sich in einem Verständnis von Wirtschaft niedergeschlagen, das stark auf Transformation und Vielfalt der Ökonomie ausgerichtet ist. Diese Vielfalt, so der Vorwurf der NES, könne die neoklassische Theorie mit ihren realitätsfernen, vereinheitlichenden Annahmen nicht adäquat abbilden. In seinem vierten Kapitel geht Sparsam dieser Kritik, die das vereinende Element der NES darstellt, auf den Grund. Sparsam moniert, dass die Realitätsferne der Neoklassik bereits innerhalb der Ökonomik aufgegriffen wurde, und schlägt stattdessen eine weitreichendere, immanente Kritik an der Neoklassik vor. Problematisch erscheint demzufolge vielmehr, dass die Ökonomik die Einheit ihres Gegenstandsbereichs, d.h. die Spezifika und gemeinsamen Grundfunktionen des kapitalistischen Wirtschaftssystems nicht zu erklären vermag. Insbesondere die Ausrichtung auf Nutzenmaximierung kann allenfalls tautologisch begründet werden. An dieser Hürde, so das weitere Argument Sparsams, scheitert jedoch auch die NES.

Ausgehend von diesem Problemaufriss ist das Kernstück der Arbeit die kritische Auseinandersetzung - man möchte hier fast von einem „Sezieren“ sprechen - mit vier zentralen Ansätzen der NES. Für das Paradigma der Netzwerktheorie sind dies Granovetters Einbettungsperspektive und Whites konstruktivis- 
tische Netzwerktheorie. Für den Neo-Institutionalismus werden der Fligstein'sche feldtheoretische Zugang zu Märkten und Beckerts neopragmatische Marktsoziologie analysiert. Für die einzelnen Theoriestränge werden Begründungszusammenhänge und Zentralmetaphern sowie die jeweils zugrundeliegende, explizite oder implizite Handlungstheorie herausgearbeitet und der Frage nachgegangen, wie der Gegenstandsbereich „Wirtschaft“ vom jeweiligen Ansatz theoretisch gefasst wird. Bei alldem werden die Ansätze einer oft beißenden, aber wohlfundierten Kritik unterzogen sowie umfassende Bezüge zur kritischen Sekundärliteratur hergestellt. Dass die Auseinandersetzung mit den einzelnen Theorien nicht in gleicher Weise systematisch erfolgt, erschwert eine vergleichende Lektüre - ist aber dem Bemühen geschuldet, den jeweiligen Spezifika gerecht zu werden. Die Befunde dieser Auseinandersetzung werden in einem Fazit verdichtet. So wird deutlich, dass die NES sich zwar von der neoklassischen Ökonomik abgrenzt, indem sie die Vorstellung einer festen Präferenzordnung und die Formalisierbarkeit als einziges Konsistenzkriterium ablehnt, gleichzeitig aber Grundannahmen ökonomischer Theorien unhinterfragt importiert. Insbesondere verzichtet auch die NES darauf, ökonomische Motive wie Profitstreben sozial zu begründen und nimmt das Primat des Marktes oder den Wert von Geld unreflektiert als gegeben an. Insgesamt konzipiert die NES das Ökonomische und das Soziale als zwar interagierende, aber doch getrennte Sphären. Worauf „das Ökonomische“ beruht, darauf weiß auch die NES keine Antwort. Diese Frage stellt, so Sparsam, das „ausgesparte Zentrum“ der NES dar. Ausgehend von seiner für die Neue Wirtschaftssoziologie wenig schmeichelhaften Diagnose zeigt Sparsam in seinem Ausblick auf, wie eine allgemeine Wirtschaftssoziologie aussehen könnte, die diesem Namen gerecht wird. Insbesondere appelliert er daran, den universalen Charakter der Wirtschaft nicht zu ignorieren und hierzu differenzierungstheoretische Gedanken partiell zu rehabilitieren.

In seiner äußerst gelungenen Kritik bringt Sparsam implizit jene Opposition in Stellung, die Boltanski und Chiapello auf die Formel Korrektivkritik vs. Radikalkritik gebracht haben (vgl. Boltanski / Chiapello, 1999). Er wirft der NES vor, zwar methodische Korrekturen einzufordern, das grundlegende System aber nicht zu hinterfragen und so letzten Endes zu dessen Stabilisierung beizutragen. Sparsams Analyse macht überzeugend deutlich, dass die NES wenig Potential hat, um die Grundpfeiler der kapitalistischen Wirtschaftsordnung in Frage zu stellen. Stattdessen begnügt sich die NES damit, die Fragestellungen der kapitalistischen Ökonomik besser, da soziologisch, zu beantworten. Die NES bietet allenfalls Reförmchen, sicher aber keine Revolution. Fraglich erscheint an Sparsams Argument lediglich, ob es sich bei diesem Anliegen nicht ebenfalls um ein legitimes Unterfangen handelt. So zielt die von der NES hervorgebrachte Korrektivkritik trotz der aufgedeckten Desiderate doch darauf ab, den Allmachtanspruch der 
neoklassischen Wirtschaftswissenschaften zu relativieren. Die NES kann, womöglich gerade aufgrund ihrer Nähe zum ökonomischen Mainstream, alternative Perspektiven salonfähig machen und so den Weg für ein breiteres Umdenken ebnen. Eine stärkere Reflexion der eigenen Leerstellen, wie Sparsam sie anmahnt, ist dafür jedoch ohne Frage dringend geboten. Die Lektüre des Bandes kann daher allen alten, neuen und werdenden Wirtschaftssoziologen nur dringend angeraten sein.

\section{Zusammenschau und Ausblick}

Welche gemeinsamen Linien und Tendenzen lassen sich aus den vorgestellten Bänden nun ableiten? Wenig überraschend positionieren sich die Autoren durchweg kritisch zur ökonomischen Neoklassik, die als Mainstream der Ökonomik ausgemacht wird. Anders als die traditionelle NES bemängeln die vorgestellten Bücher aber nicht nur die Methodik oder die realitätsfernen Annahmen wirtschaftswissenschaftlicher Ansätze. Vielmehr stellt die Kritik auch gerade auf den moralischen Gehalt dieser Annahmen sowie die implizite Legitimationsfunktion der Ökonomik ab und zielt damit weniger auf deren mangelnde „wissenschaftliche Güte“ als auf ihre gesellschaftliche Bedeutung und Wirkmacht, sprich deren Performativität.

Eine weitere gemeinsame Tendenz scheint der wiederkehrende Appell zu sein, die Ökonomik nicht leichtfertig auf die Neoklassik zu verkürzen. Stattdessen wird von den Autoren gefordert, gezielt auch heterodoxe Theorien mit einzubeziehen und kritisch zu befragen. Entlang der besprochenen Bände gelingt dies freilich erst bruchstückhaft und oft begrenzt sich die Erweiterung allenfalls auf die der Neoklassik verwandte Institutionenökonomik. Zwar gibt die Neoklassik eine wohlfeile Zielscheibe für soziologische Kritik ab - gerade eine wirtschaftssoziologische Betrachtung, die nicht implizit zur Reproduktion des neoklassischen Paradigmas beitragen will, sollte jedoch verstärkt nach alternativen Ansätzen Ausschau halten und über disziplinäre Gräben hinweg den Anschluss suchen.

Obwohl alle vorgestellten Bücher implizit oder explizit das Verhältnis zwischen Wirtschaftswissenschaften und Wirtschaft in den Blick nehmen, ist schließlich $\mathrm{zu}$ bemerken, dass nur wenige systematisch und begrifflich zwischen der Ökonomie, d.h. dem alltagsweltlichen, profanen Wirtschaften, und der Ökonomik, der sakralen Wissenschaft, unterscheiden. Allzu oft wird pauschalisierend von „der Ökonomie“ oder „dem Ökonomischen“ gesprochen. Gerade um das verquickte Verhältnis von Ökonomie und Ökonomik darzustellen und aufzuzeigen, inwiefern es den Wirtschaftswissenschaften nicht gelingt, sich von ihrem 
Untersuchungsgegenstand zu emanzipieren, bedarf es aber präziser Begrifflichkeiten. Vor dem Hintergrund dieser Begriffsfindung wird jedoch auch zu benennen sein, was „die Ökonomie“ ausmacht, wodurch sich dieser Gegenstandsbereich aus soziologischer Perspektive eingrenzen lässt. Insofern es sich bei Ökonomie, wie eingangs skizziert, jedoch um mehr handeln soll als lediglich den Gegenstand, den Wirtschaftswissenschaftler untersuchen, steht der Wirtschaftssoziologie hier noch eine spannende Debatte bevor.

\section{Literatur}

Boltanski, L.; Chiapello, E. Le nouvel esprit du capitalism; Gallimard: Paris, 1999.

Fourcade, M. Theories of markets and theories of society. American Behavioral Scientist 2007, 50, 1015-1034.

Vogl, J. Das Gespenst des Kapitals; Diaphanes: Zürich, 2010. 